\title{
Atomic-resonance-enhanced nonlinear optical frequency conversion with entangled photon pairs
}

\author{
Shengwang $\mathrm{Du}^{*}$ \\ Department of Physics, The Hong Kong University of Science and Technology, Clear Water Bay, Kowloon, Hong Kong, China
}

(Received 1 January 2011; published 7 March 2011)

\begin{abstract}
We theoretically study nonlinear optical frequency conversion with time-frequency entangled paired photons whose sum frequency is on two-photon resonance of an atomic ensemble. Assisted by a strong coupling laser, two paired photons with wide spectrum are converted into a single monochromatic photon. The on-resonance nonlinear process is made possible due to the electromagnetically induced transparency that not only eliminates the onresonance absorption but also enhances the nonlinear interaction between the single photons and atoms. Compared to this quantum-nonlinear conversion, the classical corresponding single-photon counts from accidental twophoton coincidence has a wide spectrum and experiences large absorption. As a result, the system can be used as an efficient two-photon quantum correlator in which the classical accidental coincidences can be suppressed. We perform numerical simulations basing on a $\mathrm{Rb}$ atomic vapor cell with realistic operating parameters.
\end{abstract}

DOI: 10.1103/PhysRevA.83.033807

PACS number(s): 42.50.Gy, 03.65.Ud, 42.65.-k

\section{INTRODUCTION}

The sensitivity and efficiency of entanglement-based quantum measurement always suffer from and are limited by classical correlated and uncorrelated noise. To suppress the classical noise, an entangled paired photon (or termed as biphoton) source generated from spontaneous parametric down-conversion (SPDC) [1,2] or spontaneous four-wave mixing (SFWM) [3] is usually operated at a very low output rate to avoid the accidental coincidence from uncorrelated photons. For example, photon pairs with a transform-limited waveform has a typical correlation time $1 / \Delta v$, where $\Delta v$ is the bandwidth of the biphoton joint spectrum. Therefore a SPDC source that can provide distinct photon pairs must have a generation rate less than its bandwidth. Otherwise, overlap between neighboring uncorrelated pairs generates classical coincidence counts. As a result, the conventional coincidence measurement based on the single-photon counting technique requires the photon flux of a SPDC quantum source to be less than its bandwidth, which determines the crossover between the lower power nonclassical (quantum) regime and the higher power classical regime [4]. This is the reason why many quantum-entanglement tests, such as two-photon interference [5,6], the Bell inequality test [7,8], and manyphoton entanglements $[9,10]$, take very long measurement time.

On the other hand, realizing efficient nonlinear interactions between single photons is considered a key step toward all-optical quantum-computation and quantum-information processing. There has been considerable research work on enhancing the nonlinear coupling between two photons, such as sum-frequency generation (SFG) and two-photon absorption (TPA), using entangled photon pairs because of their strong frequency-time correlation. Meanwhile, SFG and TPA can also work as fast quantum correlators to measure ultra-short time correlations between single photons whose temporal duration $(<1 \mathrm{ps})$ cannot be directly resolved by conventional single-photon counters ( $>10 \mathrm{ps}$ ) [11]. There are

\footnotetext{
*dusw@ust.hk
}

several approaches in enhancing the two-photon nonlinear interaction. The first is on-resonance enhancement using highfinesse optical cavities and narrow-linewidth atomic systems. For example, recent experiment of SFG with time-frequency entangled photons within a cavity demonstrated a resonant enhancement by a factor of 12 [4]. Resonance TPA with broadband down-converted light can be coherently controlled with high spectral and temporal resolution [11,12]. The second approach is to make biphotons with ultra-short temporal correlation length using broadband down-converted light [13]. The short temporal correlation time increases the two-photon coincidence rate when they meet inside the nonlinear medium. Many works have been focused on generating ultra-short entangled photons [14-16]. It has been shown that the quantum to classical coincidence ratio can be enhanced using SFG nonlinear crystal as a correlator for broadband entangled photons due to the narrower up-conversion bandwidth [13]. Other approaches include using tapered optical fibers to enhance TPA at low light levels $[17,18]$.

The theoretical description on the enhancement of twophoton interaction with broadband entangled photons has been given recently by Dayan [19], in good agreement with the TPA [11] and SFG [13] experiments. However, Dayan did not discuss how to enhance the material's nonlinearity, and especially limits the discussion on the cases where the dependence of the nonlinear susceptibilities on frequency is neglected. This treatment is valid for normally used nonlinear crystals and a system whose intermediate states are far away from the single-photon frequencies. It also does not account for the medium loss and absorption. In this paper, we study the sum-frequency-like nonlinear optical frequency conversion with entangled photons using electromagnetically induced transparency (EIT) in a multilevel atomic ensemble. Assisted by a strong coupling laser, two paired photons are converted into a single-monochromatic photon. The EIT not only eliminates the on-resonance absorption on the generated photon but also enhances dramatically the nonlinear interaction between the single photons and atoms. In the classical regime with two input coherent beams, the nonlinear process has been well understood [20]. In this work, we focus on the quantumnonlinear process with entangled photons, and we will discuss 
the difference between the quantum and classical cases. Our result show that the incoherent converted photons from the classical process can be significantly suppressed due to the medium loss and absorption, while the quantum generation is immune to the absorption because of EIT.

The article is organized as follows. In Sec. II, we derive the general formulism in the Heisenberg picture to describe the nonlinear frequency conversion with entangled photons from a SPDC source. In Sec. III, we discuss how to measure the two-photon correlation using the nonlinear frequency convertor. In Sec. IV, we describe the nonlinear and linear optical responses from the four-level atomic system. In Sec. V, we show that the ratio of quantum vs classical conversion can be significantly improved. In Sec. VI, we perform a numerical simulation based on a $\mathrm{Rb}$ vapor cell with realistic parameters. Finally we summarize in Sec. VII.

\section{NONLINEAR FREQUENCY CONVERSION: GENERAL FORMULISM}

The schematics of nonlinear sum-like frequency conversion with entangled photons is shown in Fig. 1. To simplify the description and also for convenience, in this paper, we illustrate the theoretical model with SPDC terms, but the results can be also applied to the SFWM photons. As shown in Fig. 1, driven by a continuous-wave (cw) monochromatic pump laser with an angular frequency $\omega_{p}$, phase-matched paired signal $\left(\omega_{s}\right)$ and idler $\left(\omega_{i}\right)$ photons are spontaneously generated from the nonlinear crystal, and then enter a four-level atomic medium. While their frequencies are far detuned from the atomic single-photon resonance to eliminate their linear propagation loss, the sum of the two frequencies is on resonance at the two-photon transition $|1\rangle \leftrightarrow|2\rangle$, that is, $\omega_{p}=\omega_{s}+\omega_{i}=\omega_{21}$. In the presence of a strong coupling laser $\left(\omega_{c}\right)$ that is on resonance of the transition $|2\rangle \leftrightarrow|3\rangle$, the paired signal-idler photons are converted into monochromatic photons $\left(\omega_{d}=\omega_{s}+\omega_{i}-\omega_{c}=\omega_{p}-\omega_{c}\right)$. The coupling laser not only enhances the nonlinear interaction due to the onresonance process but also eliminates the generated photon's on-resonance linear absorption due to the EIT effect.

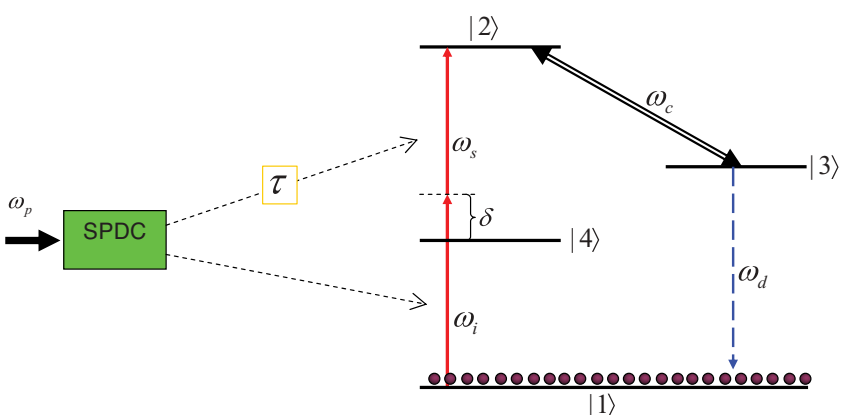

FIG. 1. (Color online) Schematics of nonlinear frequency conversion with entangled photons. With a monochromatic pump laser beam $\left(\omega_{p}\right)$, time-frequency entangled signal $\left(\omega_{s}\right)$ and idle $\left(\omega_{i}\right)$ photons are generated through spontaneous parametric down-conversion (SPDC) from a nonlinear medium (crystal), and enter a four-level atomic ensemble on two-photon resonance $\left(\omega_{s}+\omega_{i}=\omega_{21}\right)$. Driven by a strong coupling laser $\left(\omega_{c}\right)$, the two entangled photons are converted to a single photon $\left(\omega_{d}\right)$ that is on resonance with the transition $|1\rangle \rightarrow|3\rangle$.
In the Heisenberg picture where we treat the (initial) state of signal, idler, and converted photons as vacuum, the generated signal and idler fields at the output surface of the SPDC medium can be described by their field operators $\hat{a}_{s}(\omega)$ and $\hat{a}_{i}\left(\omega_{p}-\omega\right)[21]$ :

$$
\begin{gathered}
\hat{a}_{s}(\omega)=A(\omega) \hat{a}_{s 0}(\omega)+B(\omega) \hat{a}_{i 0}^{\dagger}\left(\omega_{p}-\omega\right), \\
\hat{a}_{i}^{\dagger}\left(\omega_{p}-\omega\right)=C(\omega) \hat{a}_{s 0}(\omega)+D(\omega) \hat{a}_{i 0}^{\dagger}\left(\omega_{p}-\omega\right),
\end{gathered}
$$

where $\hat{a}_{s 0}$ and $\hat{a}_{i 0}$ are their field operators at the input vacuum surface and satisfy the commutation relation $\left[\hat{a}_{m 0}(\omega), \hat{a}_{n 0}^{\dagger}\left(\omega^{\prime}\right)\right]=\delta_{m n} \delta\left(\omega-\omega^{\prime}\right)$ with $m, n$ denoting the signal and idle modes. In the initial vacuum state that does not include the strong pump and coupling classical field modes, the only nonzero expectation values are $\left\langle\hat{a}_{m 0}(\omega) \hat{a}_{n 0}^{\dagger}\left(\omega^{\prime}\right)\right\rangle=$ $\delta_{m n} \delta\left(\omega-\omega^{\prime}\right)$. At the limit of low parametric gain and without linear loss and gain inside the SPDC medium, the frequency dependent coefficients satisfy $|A(\omega)|=|D(\omega)|=1,|B(\omega)|=$ $|C(\omega)|$, and $A(\omega) C^{*}(\omega)=B(\omega) D^{*}(\omega)$. The signal and idler photon power spectrums are described by $|B(\omega)|^{2}$ and $|C(\omega)|^{2}$. The biphoton relative wave function at the Schrodinger picture can be obtained as $\Psi(\tau)=\frac{1}{2 \pi} \int d \omega A(\omega) C^{*}(\omega) e^{-i \omega \tau}$, where $\tau=t_{s}-t_{i}$ is the relative time delay between the signal and idler photons. In the following we assume $\tau=0$ or zero path difference of the signal and idler photons from the SPDC output surface to the atomic medium input surface (the effect of the relative time delay will be discussed later). In the atomic medium, their propagation effects are described by

$$
\begin{aligned}
& \hat{a}_{s}(z, t)=\frac{1}{\sqrt{2 \pi}} \int d \omega \hat{a}_{s}(\omega) e^{i\left[k_{s}(\omega) z-\omega t\right]}, \\
& \hat{a}_{i}(z, t)=\frac{1}{\sqrt{2 \pi}} \int d \omega \hat{a}_{i}(\omega) e^{i\left[k_{i}(\omega) z-\omega t\right]},
\end{aligned}
$$

where $k_{s} \simeq \omega_{s} / c$ and $k_{i} \simeq \omega_{i} / c$ are wave numbers of the signal and idle photons that are close to their values in vacuum because of the large single-photon frequency detuning $(\delta$ as indicated in Fig. 1). The converted photon field at $\omega_{d}$ is described by

$$
\hat{a}_{d}(z, t)=\frac{1}{\sqrt{2 \pi}} \int d \omega \hat{a}_{d}(z, \omega) e^{-i \omega t} .
$$

Under the slowly varying envelope approximation, $\hat{a}_{d}(z, \omega)$ is governed by

$$
\begin{aligned}
& {\left[\frac{\partial}{\partial z}-i k_{d}\left(\omega_{d}\right)\right] \hat{a}_{d}\left(z, \omega_{d}\right)} \\
& =\hat{F}+\frac{i \beta}{\sqrt{2 \pi}} \int d \omega_{s} e^{i\left(k_{i}+k_{s}-k_{c}\right) z} \chi^{(3)}\left(\omega_{s}, \omega_{d}+\omega_{c}-\omega_{s}, \omega_{d}\right) \\
& \quad \times \hat{a}_{s}\left(\omega_{s}\right) \hat{a}_{i}\left(\omega_{d}+\omega_{c}-\omega_{s}\right),
\end{aligned}
$$

where $k_{d}=\frac{\omega_{d}}{c} \sqrt{1+\chi}$ is the complex wave number with the EIT linear susceptibility $\chi$. Here for simplification, we have assumed all involved fields are single mode and propagate collinearly along the $z$ direction. $\beta=$ $\left[E_{c}^{*} /(2 c)\right] \sqrt{2 \hbar \varpi_{s} \varpi_{i} \varpi_{d} A_{d} /\left(c \epsilon_{0} A_{s} A_{i}\right)}$ is a grouped constant where $E_{c}^{*}$ is the conjugate of the coupling laser field envelope, $c$ is the speed of light in vacuum, $\varpi_{m}(m=s, i$, and $d)$ are central angular frequencies of signal, idle, and converted photons, respectively, $A_{m}$ are single-mode transverse 
cross-section areas, and $\epsilon_{0}$ is the vacuum dielectric constant. $\chi^{(3)}$ is the third-order nonlinear susceptibility. $\hat{F}$ is the Langevin noise operator that preserves the commutation relation of the field operators. It can be shown that, under the ground state approximation that the atomic population remains mainly at the ground state $|1\rangle$, the Langevin noise operator does not contribute to the conversion frequency generation. Therefore, from now on, we ignore the Langevin noise operator. to be

The solution to Eq. (4) at the output surface $z=L$ is found

$$
\begin{aligned}
\hat{a}_{d}\left(L, \omega_{d}\right)= & \hat{a}_{d 0} e^{i k_{d} L}+\frac{i \beta L}{\sqrt{2 \pi}} \int d \omega_{s} \chi^{(3)}\left(\omega_{s}, \omega_{d}+\omega_{c}-\omega_{s}, \omega_{d}\right) \\
& \times \Phi(\Delta k) \hat{a}_{s}\left(\omega_{s}\right) \hat{a}_{i}\left(\omega_{d}+\omega_{c}-\omega_{s}\right),
\end{aligned}
$$

where the first term is the vacuum field propagation and does not contribute to the generated photons. The linear propagation loss and phase-mismatching factor are grouped into the $\Phi$ function

$$
\Phi(\Delta k)=\operatorname{sinc}(\Delta k L / 2) e^{-i \Delta k L / 2} e^{i k_{d} L},
$$

with $\Delta k=k_{d}+k_{c}-k_{s}-k_{i}$ and $\operatorname{sinc}(x)=\sin (x) / x$. The mathematic form of solution (5) is similar to that of SFG [19], but the rich physics will be discussed in this paper on how to control the nonlinear frequency conversion through manipulating the linear and nonlinear susceptibilities $(\chi$ and $\left.\chi^{(3)}\right)$.

The field autocorrelation of the generated photons can be obtained by

$$
\begin{aligned}
C_{d}(\Delta t)= & \left\langle\hat{a}_{d}^{\dagger}(L, t) \hat{a}_{d}(L, t+\Delta t)\right\rangle \\
= & \frac{1}{2 \pi} \iint d \omega_{d} d \omega_{d}^{\prime} e^{-i \omega_{d} t} e^{-i \omega_{d}^{\prime}(t+\Delta t)}\left\langle\hat{a}_{d}^{\dagger}\left(L, \omega_{d}\right) \hat{a}_{d}\left(L, \omega_{d}^{\prime}\right)\right\rangle \\
= & \left(\frac{\beta L}{2 \pi}\right)^{2}\left|\int d \omega_{s} \chi^{(3)}\left(\omega_{s}, \omega_{p}-\omega_{s}, \omega_{p}-\omega_{c}\right) \Phi(\Delta k) A\left(\omega_{s}\right) C^{*}\left(\omega_{s}\right)\right|^{2} e^{-i\left(\omega_{p}-\omega_{c}\right) \Delta t} \\
& +\left(\frac{\beta L}{2 \pi}\right)^{2} \iint d \omega_{s} d \omega_{i}\left|\chi^{(3)}\left(\omega_{s}, \omega_{i}, \omega_{s}+\omega_{i}-\omega_{c}\right) \Phi(\Delta k) B\left(\omega_{s}\right) C\left(\omega_{p}-\omega_{i}\right)\right|^{2} e^{-i\left(\omega_{s}+\omega_{i}-\omega_{c}\right) \Delta t} .
\end{aligned}
$$

We can obtain the power spectrum from the inverse Fourier transform of the field autocorrelation function

$$
\begin{aligned}
P_{d}(\omega)= & \int d \Delta t C_{d}(\tau) e^{i \omega \Delta t} \\
= & \frac{\beta^{2} L^{2}}{2 \pi}\left|\int d \omega_{s} \chi^{(3)}\left(\omega_{s}, \omega_{p}-\omega_{s}, \omega_{p}-\omega_{c}\right) \Phi(\Delta k) A\left(\omega_{s}\right) C^{*}\left(\omega_{s}\right)\right|^{2} \delta\left(\omega+\omega_{c}-\omega_{p}\right) \\
& +\frac{\beta^{2} L^{2}}{2 \pi} \int d \omega_{s}\left|\chi^{(3)}\left(\omega_{s}, \omega+\omega_{c}-\omega_{s}, \omega\right) \Phi(\Delta k) B\left(\omega_{s}\right) C\left(\omega_{p}+\omega_{s}-\omega_{c}-\omega\right)\right|^{2} .
\end{aligned}
$$

There are two terms in the field autocorrelation function (7) and power spectrum (8): the first corresponds to the quantum conversion from the frequency entanglement of the signal and idle photons that lead to generation of the converted photon at monochromatic frequency of $\omega_{p}-\omega_{c}$, and the second is the classical term with a wider spectrum resulting from the sum of the independent frequency components of the signal and idle photons as a form of convolution. The single-photon generation rate at the converted frequency can be obtained from the field autocorrelation at $\tau=0$ :

$$
\begin{aligned}
R_{d}= & \left\langle\hat{a}_{d}^{\dagger}(L, t) \hat{a}_{d}(L, t)\right\rangle=C_{d}(0) \\
= & \left(\frac{\beta L}{2 \pi}\right)^{2}\left|\int d \omega_{s} \chi^{(3)}\left(\omega_{s}, \omega_{p}-\omega_{s}, \omega_{p}-\omega_{c}\right) \Phi(\Delta k) A\left(\omega_{s}\right) C^{*}\left(\omega_{s}\right)\right|^{2} \\
& +\left(\frac{\beta L}{2 \pi}\right)^{2} \iint d \omega_{s} d \omega_{i}\left|\chi^{(3)}\left(\omega_{s}, \omega_{i}, \omega_{s}+\omega_{i}-\omega_{c}\right) \Phi(\Delta k) B\left(\omega_{s}\right) C\left(\omega_{p}-\omega_{i}\right)\right|^{2},
\end{aligned}
$$

which is also contributed from both the quantum-frequency entanglement (the first term) and classical accidental coincidence (the second term).

\section{TWO-PHOTON CORRELATION}

In the following we discuss the possibility of using the nonlinear frequency conversion to measure the two-photon correlation that enhances the quantum generation and suppresses the classical correlation. To do so, we first describe how the two-photon Glauber correlation is measured by two single-photon counters:

$$
\begin{aligned}
G_{s i}^{(2)}(\tau)= & \left\langle\hat{a}_{i}^{\dagger}(t) \hat{a}_{s}^{\dagger}(t+\tau) \hat{a}_{s}(t+\tau) \hat{a}_{i}(t)\right\rangle \\
= & \frac{1}{(2 \pi)^{2}}\left|\int d \omega_{s} A\left(\omega_{s}\right) C^{*}\left(\omega_{s}\right) e^{-i \omega_{s} \tau}\right|^{2} \\
& +\frac{1}{(2 \pi)^{2}} \iint d \omega_{s} d \omega_{i}\left|B\left(\omega_{s}\right) C\left(\omega_{p}-\omega_{i}\right)\right|^{2} \\
= & |\Psi(\tau)|^{2}+N_{s} N_{i},
\end{aligned}
$$


where $|\Psi(\tau)|^{2}$ is a modular square of the biphoton wave packet. In the second term, $N_{s}=N_{i}=N_{0}$ are signal and idler singlephoton generation rates and contribute to uncorrelated noise background. The normalized two-photon correlation function is

$$
\begin{aligned}
g_{s i}^{(2)}(\tau) & =\frac{\left\langle\hat{a}_{i}^{\dagger}(t) \hat{a}_{s}^{\dagger}(t+\tau) \hat{a}_{s}(t+\tau) \hat{a}_{i}(t)\right\rangle}{\left\langle\hat{a}_{i}^{\dagger}(t) \hat{a}_{i}(t)\right\rangle\left\langle\hat{a}_{s}^{\dagger}(t) \hat{a}_{s}(t)\right\rangle} \\
& =1+\frac{|\Psi(\tau)|^{2}}{N_{s} N_{i}} .
\end{aligned}
$$

It is clear that the nonclassical regime holds $|\psi(\tau)|^{2}>N_{s} N_{i}$, which is required to violate the Cauchy-Schwartz inequality [22].

To measure the signal and idler two-photon correlation with the nonlinear frequency conversion, we introduce a relative time delay $\tau$ in the signal photon channel before entering the atomic medium. Equivalently, we have $A^{\prime}(\omega)=A(\omega) e^{-i \omega \tau}$ and $B^{\prime}(\omega)=B(\omega) e^{-i \omega \tau}$. The single-photon generation rate at the converted frequency becomes

$$
\begin{aligned}
R_{d}(\tau)= & \left\langle\hat{a}_{d}^{\dagger}(L, t ; \tau) \hat{a}_{d}(L, t ; \tau)\right\rangle \\
= & \left(\frac{\beta L}{2 \pi}\right)^{2}\left|\int d \omega_{s} \chi^{(3)}\left(\omega_{s}, \omega_{p}-\omega_{s}, \omega_{p}-\omega_{c}\right) \Phi(\Delta k) A\left(\omega_{s}\right) C^{*}\left(\omega_{s}\right) e^{-i \omega_{s} \tau}\right|^{2} \\
& +\left(\frac{\beta L}{2 \pi}\right)^{2} \iint d \omega_{s} d \omega_{i}\left|\chi^{(3)}\left(\omega_{s}, \omega_{i}, \omega_{s}+\omega_{i}-\omega_{c}\right) \Phi(\Delta k) B\left(\omega_{s}\right) C\left(\omega_{p}-\omega_{i}\right)\right|^{2} \\
= & \beta^{2} L^{2}|\widetilde{\Psi}(\tau)|^{2}+\beta^{2} L^{2} \widetilde{N}_{s i} .
\end{aligned}
$$

The first term in Eq. (12) corresponds to the entangled photon correlation and the second term to the uncorrelated background noise floor. As we know the linear and nonlinear susceptibilities of the medium, the original biphoton correlation function $|\Psi(\tau)|^{2}$ can be reconstructed from the modified correlation function $|\widetilde{\Psi}(\tau)|^{2}$. On the other side, due to the EIT narrow transparency window and phase-matching condition, the uncorrelated two-photon coincidence counts $\widetilde{N}_{s i}$ can be significantly suppressed because of its wide spectrum. Therefore, the quantum-classical ratio can be increased by many orders compared to the conventional SPC technique, as shown in the later numerical simulation.

To characterize the enhancement of the ratio of quantumcorrelation signal to classical-uncorrelated noise, we define a new normalized-correlation function as the following:

$$
\widetilde{g}_{s i}^{(2)}(\tau)=\frac{R_{d}(\tau)}{\beta^{2} L^{2} \widetilde{N}_{s i}}=1+\frac{|\widetilde{\Psi}(\tau)|^{2}}{\widetilde{N}_{s i}} .
$$

\section{ATOMIC NONLINEAR AND LINEAR OPTICAL RESPONSES}

As discussed in the previous sections, the converted photon generation is fully characterized by the medium nonlinear response $\left(\chi^{(3)}\right)$ and linear propagation effect $(\Phi)$. EIT plays important roles on both sides. The third-order nonlinear susceptibility describes the nonlinear interaction strength between the optical fields and atoms locally. By eliminating or reducing the on-resonance absorption loss, we can obtain unusual huge optical nonlinearity near atomic resonance. On the other hand, the nonlinear frequency conversion from the atoms at different positions have different phases due to the wave nature of the relevant fields. As a result, the EIT linear susceptibility of the generated field determines the phase matching and transmission bandwidth.

For the four-level atomic system illustrated in Fig. 1, the third-order nonlinear susceptibility of generating the converted photon is [20]

$$
\begin{aligned}
\chi^{(3)}\left(\omega_{s}, \omega_{i}, \omega_{s}+\omega_{i}-\omega_{c}\right) & =\frac{N \mu_{13} \mu_{32} \mu_{24} \mu_{41} /\left(\varepsilon_{0} \hbar^{3}\right)}{\left(\omega_{i}-\omega_{41}+i \gamma_{14}\right)\left[\left|\Omega_{c}\right|^{2}-4\left(\omega_{s}+\omega_{i}-\omega_{21}+i \gamma_{13}\right)\left(\omega_{s}+\omega_{i}-\omega_{21}+i \gamma_{12}\right)\right]} \\
& =\chi_{0}^{(3)} \frac{\left|\Omega_{c}\right|^{2}+4 \gamma_{13} \gamma_{12}}{\left[\left|\Omega_{c}\right|^{2}-4\left(\omega_{s}+\omega_{i}-\omega_{21}+i \gamma_{13}\right)\left(\omega_{s}+\omega_{i}-\omega_{21}+i \gamma_{12}\right)\right]},
\end{aligned}
$$

where $N$ is the atomic density, $\mu_{i j}$ is the electric dipole matrix element between the states $|i\rangle$ and $|j\rangle, \omega_{i j}$ is the on-resonance optical angular frequency of the transition $|i\rangle \rightarrow|j\rangle$, and $\gamma_{i j}$ is the dephasing rate between $|i\rangle$ and $|j\rangle$. On the condition of twophoton resonance $\omega_{s}+\omega_{i}=\omega_{21}$, the nonlinear susceptibility reduces to

$$
\chi_{0}^{(3)}=\frac{N \mu_{13} \mu_{32} \mu_{24} \mu_{41} /\left(\varepsilon_{0} \hbar^{3}\right)}{\left(\omega_{i}-\omega_{41}+i \gamma_{14}\right)\left(\left|\Omega_{c}\right|^{2}+4 \gamma_{13} \gamma_{12}\right)} .
$$

From Eqs. (14) and (15), we find that in the case where the signal and idler photons are far detuned from the single-photon transition, that is, $|\delta|=\left|\omega_{i}-\omega_{41}\right| \gg \gamma_{14}, \chi_{0}^{(0)}$ is nearly a constant. As a result, when the down-conversion bandwidth of the signal and idler photons is smaller than their single-photon detuning, for the quantum generation with perfectly matched signal and frequencies $\left(\omega_{s}+\omega_{i}-\omega_{21}=0\right)$, the nonlinear susceptibility is nearly frequency independent. When the 
two-photon detuning is large $\left(\omega_{s}+\omega_{i}-\omega_{21} \gg \Omega_{c}\right)$, the nonlinear interaction becomes weak.

On the other side, the down-converted photons $\left(\omega_{d}\right)$ are on the transition $|1\rangle \rightarrow|3\rangle$ within a ladder-type EIT system. In this paper we take the ground-state approximation where the atoms are mainly populated at the ground level $|1\rangle$, the EIT linear susceptibility can be obtained as

$$
\chi_{d}\left(\omega_{d}\right)=\frac{4 N\left|\mu_{13}\right|^{2}\left(\omega_{d}-\omega_{31}+i \gamma_{12}\right) /\left(\varepsilon_{0} \hbar\right)}{\left|\Omega_{c}\right|^{2}-4\left(\omega_{d}-\omega_{31}+i \gamma_{13}\right)\left(\omega_{d}-\omega_{31}+i \gamma_{12}\right)} .
$$

The signal and idler photon linear susceptibilities are

$$
\chi_{s, i}=\frac{-N\left|\mu_{14}\right|^{2} /\left(\varepsilon_{0} \hbar\right)}{\omega_{s, i}-\omega_{41}-i \gamma_{14}} \simeq \frac{-N\left|\mu_{14}\right|^{2} /\left(\varepsilon_{0} \hbar\right)}{\omega_{s, i}-\omega_{41}} .
$$

At the limit of large single-photon detuning of signal and idler photons, Eq. (6) can be approximated as

$$
\begin{aligned}
\Phi \simeq & \operatorname{sinc}\left[\frac{\delta_{s i} L}{2 V_{g}}+i \frac{\alpha\left(\delta_{s i}\right) L}{2}\right] \exp \left[i \frac{\delta_{s i} L}{2 V_{g}}-\frac{\alpha\left(\delta_{s i}\right) L}{2}\right] \\
& \times \exp \left(i k_{d 0} L\right),
\end{aligned}
$$

where $\delta_{s i}=\omega_{s}+\omega_{i}-\omega_{21}$ is the two-photon detuning, $V_{g}$ is the slow-light group velocity induced by EIT, $\alpha\left(\delta_{s i}\right)$ is the linear absorption coefficient caused by $\gamma_{12}$ [3], and $k_{d 0}$ is the wave vector number of the generated field in vacuum. Equation (18) reveals two important bandwidths caused by the linear propagation effect: the slow light induced phasematching bandwidth $\Delta \omega_{g}=2 \pi V_{g} / L$ and EIT transparency bandwidth $\Delta \omega_{e} \simeq\left|\Omega_{c}\right|^{2} /\left[2 \gamma_{13} \sqrt{\alpha(0) L}\right]$ [3].

\section{QUANTUM VS CLASSICAL}

There are three characteristic frequencies that determine the medium nonlinear frequency conversion efficiency. The first is the coupling laser Rabi frequency $\Omega_{c}$ that determines the nonlinear interaction bandwidth from $\chi^{(3)}$. The second is the EIT transparency window bandwidth $\Delta \omega_{e}$. The third is the phase-matching bandwidth $\Delta \omega_{g}$. The entire nonlinear conversion bandwidth is then determined by $\min \left\{\Omega_{c}, \Delta \omega_{e}, \Delta \omega_{g}\right\}$. With a monochromatic pump laser $\left(\omega_{p}\right)$, the converted photons from frequency entanglement $\left(\omega_{s}+\omega_{i}=\omega_{c}\right)$ are also monochromatic $\left(\omega_{d}=\omega_{p}-\omega_{c}\right)$. As a result, the quantum generation reaches the maximum efficiency. On the other side, the classical generation is highly suppressed due to the wide two-photon bandwidth $\delta_{s i} \gg\left\{\Omega_{c}, \Delta \omega_{e}, \Delta \omega_{g}\right\}$.

For the quantum generation of converted photons, we have $\delta_{s i}=0$ for all signal-idler frequency components that lead to monochromatic frequency at $\omega_{d}=\omega_{p}-\omega_{c}$ and on resonance to the EIT transition. When the signal and idler singlephoton detuning is larger than their bandwidth $\left(\omega_{s, i}-\omega_{41} \gg\right.$ $2 \pi \times \Delta \nu)$, the nonlinear susceptibility approaches $\chi_{0}^{(3)}$ in Eq. (15). Meanwhile the linear propagation function becomes $\Phi_{0} \simeq \operatorname{sinc}\left[i \frac{\alpha(0) L}{2}\right] \exp \left[-\frac{\alpha(0) L}{2}\right] \exp \left(i k_{d 0} L\right)$ which approaches $\exp \left(i k_{d 0} L\right)$ under the ideal EIT condition. With a biphoton transform-limited waveform, we have approximately $\Psi(0) \simeq$ $\sqrt{N_{0} \Delta v}$, where $N_{0}$ is the paired photon generation rate from the SPDC source, and estimate the quantum-generation term (at $\tau=0$ ) from Eq. (12) as

$$
R_{q} \simeq\left|\frac{\beta L}{2 \pi} \chi_{0}^{(3)} \Phi_{0}\right|^{2} N_{0} \Delta \nu
$$

On the other side for the classical generation of converted photons, the two-photon detuning $\delta_{s i}$ has a wide distribution with a bandwidth of $\sqrt{2} \Delta v$. The classical generation term (at $\tau=0$ ) from Eq. (12) can be approximated as

$$
R_{c} \simeq\left|\frac{\beta L}{2 \pi} \bar{\chi}^{(3)} \bar{\Phi}\right|^{2} N_{0}^{2},
$$

where $\bar{\chi}^{(3)}$ and $\bar{\Phi}$ are the effective nonlinear susceptibility and longitudinal function over the wide spectrum. As a result, the quantum vs classical ratio (QCR) can be expressed as

$$
\mathrm{QCR}=\frac{|\widetilde{\Psi}(0)|^{2}}{\widetilde{N}_{s i}}=\frac{R_{q}}{R_{c}} \simeq\left|\frac{\chi_{0}^{(3)} \Phi_{0}}{\bar{\chi}^{(3)} \bar{\Phi}}\right|^{2} \frac{\Delta v}{N_{0}},
$$

which also determines the visibility of the new normalized correlation function (13). If we measure the two-photon coincidence using electronic single-photon counters, this ratio becomes $\Delta v / N_{0}$ which limit the photon pair flux to be $N_{0} \leqslant$ $\Delta v$. However, in the EIT-assisted four-level atomic system discussed in the paper, in order to have the quantum generation dominate, the maximum photon pair flux can be $\left|\frac{\chi_{0}^{(3)} \Phi_{0}}{\bar{\chi}^{(3)} \Phi}\right|^{2} \Delta v$. As shown in the later simulation results, the enhancement factor of $\left|\frac{\chi_{0}^{(3)} \Phi_{0}}{\bar{\chi}^{(3)} \bar{\Phi}}\right|^{2}$ caused by EIT nonlinear susceptibility and phase matching can be as high as $10^{6}$.

\section{NUMERICAL SIMULATION}

To verify the theory, we take a $\mathrm{Rb}$ vapor cell with realistic parameters for numerical simulations. Figure 2 shows the relevant energy levels. The $\mathrm{Rb}$ cell has a length of $L=1 \mathrm{~cm}$ and is heated up to $155^{\circ} \mathrm{C}$ to obtain a high density $N=$ $10^{20} \mathrm{~m}^{-3}$. Here we take degenerate signal and idle photons at $778 \mathrm{~nm}$ whose sum frequency coincides with the twophoton resonance transition $|1\rangle \rightarrow|2\rangle$. Because of the large

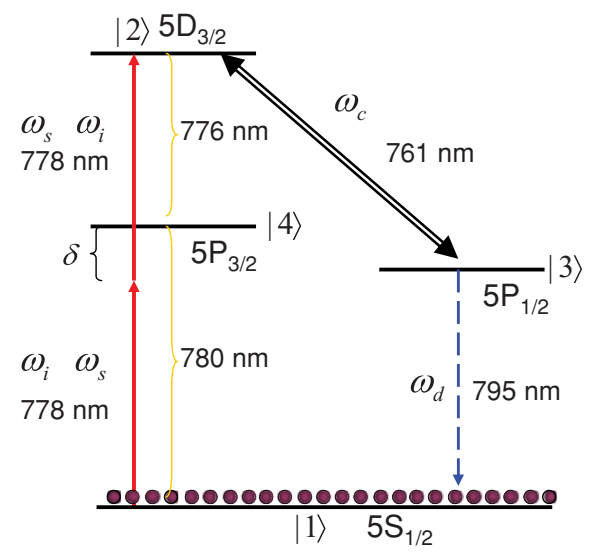

FIG. 2. (Color online) $\mathrm{Rb}$ energy level diagram for numerical simulation with degenerate signal and idle photons at $778 \mathrm{~nm}$. The coupling laser is at $761 \mathrm{~nm}$. The generated photons are at $795 \mathrm{~nm}$. 
single-photon detuning $\delta$, we can work with the fine structure without accounting for the hyperfine splitting. The energy level configuration takes $|1\rangle=\left|5 S_{1 / 2}\right\rangle,|2\rangle=\left|5 D_{3 / 2}\right\rangle,|3\rangle=$ $\left|5 P_{1 / 2}\right\rangle$, and $|4\rangle=\left|5 P_{3 / 2}\right\rangle$. The coupling laser is at 761 $\mathrm{nm}$ and the generated photons are at $795 \mathrm{~nm}$. All four fields propagate collinearly in the same direction and are focused at the center of the $\mathrm{Rb}$ cell with a waist radius $\left(1 / e^{2}\right.$ intensity) of $35 \mu \mathrm{m}$. Other relevant system parameters are $\mu_{13}=\mu_{41}=1.50 \times 10^{-29} \mathrm{C} \mathrm{m}, \mu_{32}=4.23 \times 10^{-29} \mathrm{C}$ $\mathrm{m}, \mu_{24}=1.34 \times 10^{-29} \mathrm{C} \mathrm{m}, \gamma_{14}=\gamma_{13}=2 \pi \times 3 \mathrm{MHz}$, and $\gamma_{12}=2 \pi \times 0.35 \mathrm{MHz}$. To overcome the Doppler broadening, we take $\Omega_{c}=1000 \gamma_{13}$ that corresponds to a coupling laser power of about $1.1 \mathrm{~W}$ that is commercially available.

We simulate the nonlinear frequency conversion from a SPDC source that generate paired photons at a rate of $N_{0}=10^{12}$ pairs $/ \mathrm{s}$. The single photons have a Gaussian power spectrum with a a full width of half maximum (FWHM) of $\Delta v=1 \mathrm{THz}$. The atoms at $155^{\circ} \mathrm{C}$ follow the MaxwellBoltzmann velocity distribution. Figure 3(a) displays the EIT transmission profile (dashed curve) and phase matching spectrum $\left(|\Phi|^{2}\right.$, solid curve) as functions of the two-photon detuning $\Delta \omega_{d}$. The EIT has a peak transmission of $63 \%$ and a FWHM bandwidth of $0.32 \mathrm{GHz}$. The EIT resonance loss is caused by the finite lifetime of the level $|3\rangle$ and Doppler effect. The narrow phase matching spectrum (FWHM $0.04 \mathrm{GHz}$ ) sits within the EIT window. Therefore the monochromatic quantum generation at $\Delta \omega_{d}=0$, whose bandwidth is determined by the pump laser linewidth and can be as narrow as $<1.0 \mathrm{MHz}$, sees maximum transmission and generation efficiency. For comparison, we plot the wide single-photon
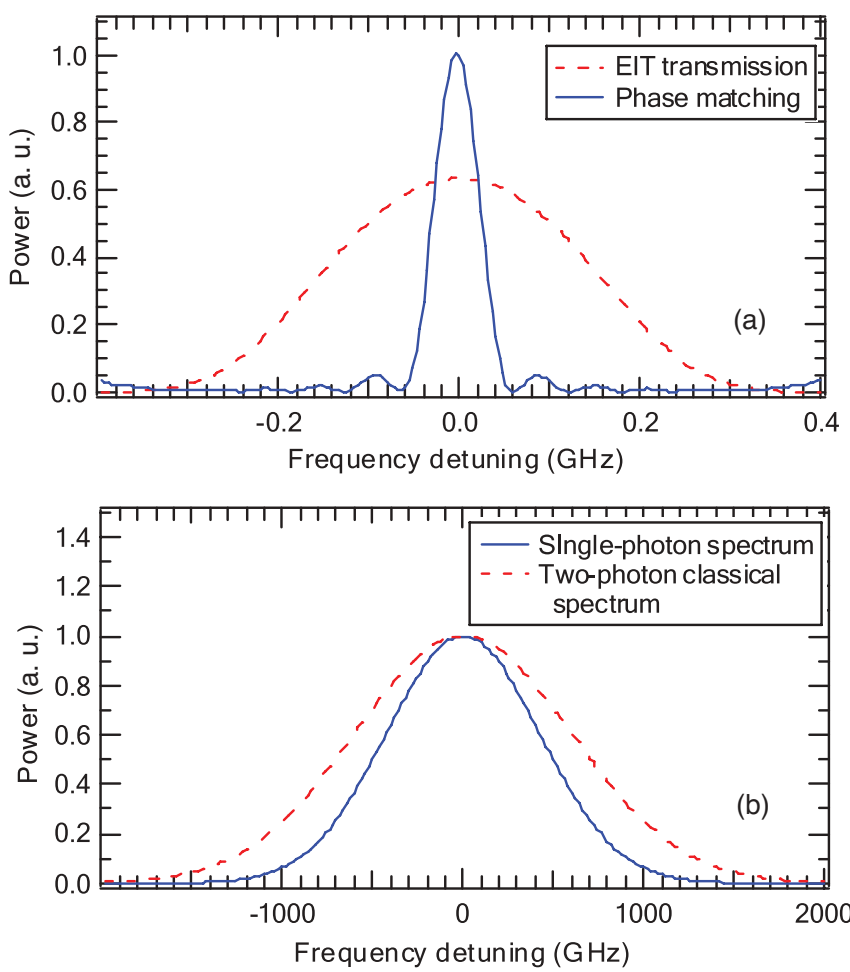

FIG. 3. (Color online) (a) EIT transmission and phase matching spectrum and (b) single-photon and two-photon classical spectrum. The single-photon power spectrum has a Gaussian shape with a FWHM bandwidth of $1 \mathrm{THz}$.
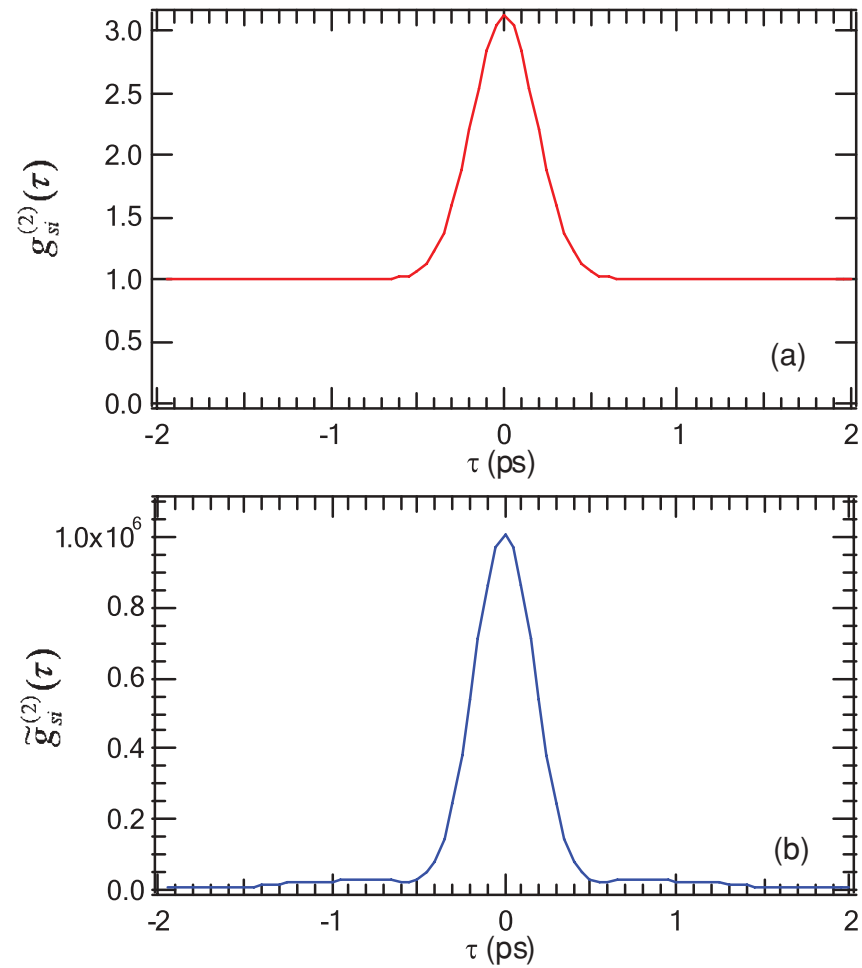

FIG. 4. (Color online) Normalized two-photon correlation functions measured by (a) ultra-fast electronic single-photon counters and (b) the proposed nonlinear frequency convertor. The single-photon power spectrum has a Gaussian shape with a FWHM bandwidth of $1 \mathrm{THz}$. The SPDC source is operated at an output rate of $10^{12}$ pairs $/ \mathrm{s}$.

spectrum (solid curve) in Fig. 3(b). The classical two-photon joint spectrum is plotted as the dashed curve in Fig. 3(b) and has a wider spectrum with a bandwidth of about $1.4 \mathrm{THz}$. As a result, the classical contribution to the frequency conversion is significantly suppressed due to the near-resonance absorption and phase mismatching. To further confirm the result, we plot

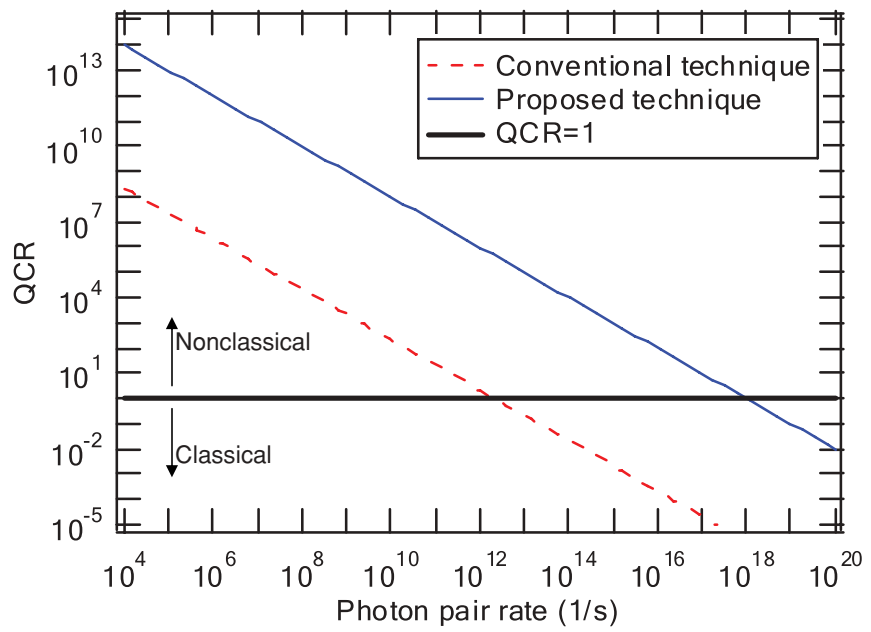

FIG. 5. (Color online) Quantum-to-classical ratio (QCR) of the two-photon correlation as a function of the photon pair rate. The dashed curve is for using the conventional single-photon counters and the solid curve for using the proposed technique. 

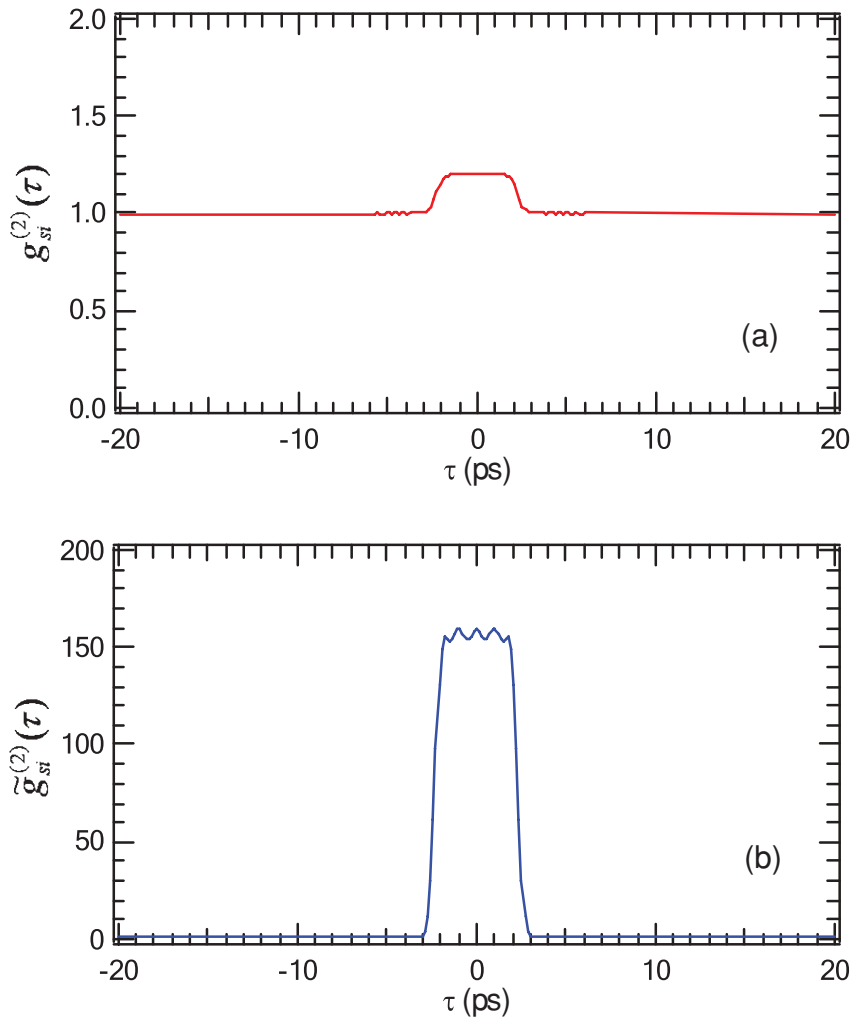

FIG. 6. (Color online) Square-shaped normalized two-photon correlation functions measured by (a) ultra-fast electronic singlephoton counters and (b) the proposed nonlinear frequency convertor. The photons have a correlation time length of $5 \mathrm{ps}$ and bandwidth of $200 \mathrm{GHz}$. The SPDC source is operated at a generation rate of $10^{12}$ pairs $/ \mathrm{s}$.

the normalized two-photon correlation function in Fig. 4. In the case of using two ultra-fast electronic single-photon counters [molded by Eq. (11)], because of the classical accidental coincidence floor [as shown in Fig. 4(a)], the transform-limited correlation function peaks at 3 with a visibility of only $50 \%$ that barely violates the Cauchy-Schwartz inequality [22]. If we use the proposed nonlinear frequency convertor to measure the correlation, due to the suppression of the classical noncoherent generation, we obtain the $\widetilde{g}_{s i}^{(2)}(\tau)$ with a peak as high as $10^{6}$ that corresponds to a visibility of nearly $100 \%$. The Gaussian profile of the two-photon waveform is well resolved. Under the above parameters, the system generate about 5000 photons/s at $\omega_{d}$ at $\tau=0$, while the classical generation is only about 0.005 photons / s that contributes to the uncorrelated floor.

We are interested in the regime where the quantum generation dominates. Figure 5 shows the QCR as a function of photon pair rate $\left(N_{0}\right)$. It is obvious that the QCR drops as $N_{0}$ increases. In the conventional single-photon counting regime, as expected, QCR becomes $<1$ when the photon pair rate is higher than the SPDC bandwidth $\left(N_{0}>\Delta v\right)$. In using the proposed technique, this limit moves to $10^{18} / \mathrm{s}$ and the QCR is enhanced by a factor of $10^{6}$.

To demonstrate that the nonlinear frequency convertor can work as an ultra-fast two-photon correlator for paired photons with an arbitrary waveform, we next consider the SPDC source with square-shape correlation. The simulation results, from the SPDC biphoton source with a correlation time length of $5 \mathrm{ps}$ at a generation rate of $N_{0}=10^{12}$, are shown in Fig. 6. In the conventional single-photon counting technique, $g_{s i}^{(2)}(0)$ is only about 1.2 . Since the pair rate is higher than the SPDC bandwidth, that is, $N_{0}>\Delta v$, the QCR $=0.2<1$ and the classical accidental coincidence dominates. This is often referred to as the classical regime. If we use the nonlinear frequency convertor to measure the correlation [as shown in Fig. 6(b)], the $\widetilde{g}_{s i}^{(2)}(0)$ increases to about 150 contributed from the time-frequency entanglement while the square shape is well resolved. We obtain QCR $=149$ that is significantly higher than the conventional technique. The photon generation rate at $\omega_{d}$ is $300 / \mathrm{s}$ in this case.

\section{SUMMARY}

In summary, we have studied the quantum theory of nonlinear optical frequency conversion with time-frequency entangled paired photons in a four-level atomic ensemble driven by a strong coupling laser. The EIT effect plays two important roles in generating the converted photons: (1) it increases the nonlinear interaction between photons and atoms with resonance enhancement by eliminating or reducing the on-resonance absorption and (2) the EIT-induced phase matching and transmission determine a very narrow conversion bandwidth. As a result, the quantum generation from the time-frequency entanglement is monochromatic and has a maximum conversion efficiency, while the classical generation from accidental two-photon coincidence has a wide bandwidth and is suppressed dramatically. These conclusions are verified by numerical simulations using a hot $\mathrm{Rb}$ vapor cell with realistic parameters. We obtain a QCR as high as $10^{6}$ and show that the nonclassical nature of the biphtons can survive and be measured at a flux rate that is much higher than the photon bandwidth. Our results also show that the nonlinear optical frequency convertor can work as an efficient and fast two-photon correlator. The proposed technique may find application in entanglement-based quantum measurements with high sensitivity.

\section{ACKNOWLEDGMENT}

The work was supported by a grant from the Research Grants Council of the Hong Kong Special Administrative Region, China (Project No. HKUST600809).
[1] S. E. Harris, M. K. Oshman, and R. L. Byer, Phys. Rev. Lett. 18, 732 (1967).

[2] D. Burnham and D. Weinberg, Phys. Rev. Lett. 25, 84 (1970).
[3] S. Du, J. Wen, and M. H. Rubin, J. Opt. Soc. Am. B 25, C98 (2008).

[4] S. Sensarn, I. Ali-Khan, G. Y. Yin, and S. E. Harris, Phys. Rev. Lett. 102, 053602 (2009). 
[5] C. K. Hong, Z. Y. Ou, and L. Mandel, Phys. Rev. Lett. 59, 2044 (1987).

[6] Y. H. Shih and C. O. Alley, Phys. Rev. Lett. 61, 2921 (1988).

[7] J. S. Bell, Physics 1, 195 (1965).

[8] A. Aspect, P. Grangier, and G. Roger, Phys. Rev. Lett. 47, 460 (1981).

[9] C.-Y. Lu, X.-Q. Zhou, O. Guhne, W.-B. Gao, J. Zhang, Z.-S. Yuan, A. Goebel, T. Yang, and J.-W. Pan, Nature Phys. 3, 91 (2007).

[10] Z. Zhao, Y.-A. Chen, A.-N. Zhang, T. Yang, H. J. Briegel, and J.-W. Pan, Nature (London) 430, 55 (2004).

[11] B. Dayan, A. Pe'er, A. A. Friesem, and Y. Silberberg, Phys. Rev. Lett. 93, 023005 (2004).

[12] N. Ph. Georgiades, E. S. Polzik, K. Edamatsu, H. J. Kimble, and A. S. Parkins, Phys. Rev. Lett. 75, 3426 (1995).
[13] B. Dayan, A. Pe'er, A. A. Friesem, and Y. Silberberg, Phys. Rev. Lett. 94, 043602 (2005).

[14] S. E. Harris, Phys. Rev. Lett. 98, 063602 (2007).

[15] S. Sensarn, G. Y. Yin, and S. E. Harris, Phys. Rev. Lett. 104, 253602 (2010).

[16] M. B. Nasr, S. Carrasco, B. E. A. Saleh, A. V. Sergienko, M. C. Teich, J. P. Torres, L. Torner, D. S. Hum, and M. M. Fejer, Phys. Rev. Lett. 100, 183601 (2008).

[17] H. You, S. M. Hendrickson, and J. D. Franson, Phys. Rev. A 80, 043823 (2009).

[18] S. M. Hendrickson, M. M. Lai, T. B. Pittman, and J. D. Franson, Phys. Rev. Lett. 105, 173602 (2010).

[19] B. Dayan, Phys. Rev. A 76, 043813 (2007).

[20] S. E. Harris, J. E. Field, and A. Imamoglu, Phys. Rev. Lett. 64, 1107 (1990).

[21] S. E. Harris, Phys. Rev. A 78, 021807(R) (2008).

[22] J. F. Clauser, Phys. Rev. D 9, 853 (1974). 